XI.

\title{
Die Thätigkeit der Ansiedelungskommission für die Provinzen Westpreufsen und Posen im Jahre 1896.
}

Auf Grund der an den Landtag der preufsischen Monarchie eingereichten Denkschrift zusanmengestellt von Ur. W. Schultze.

In zwoiten Halbband des Jahrganges 1896 dieser Jahrbüchor hatton wjr über die Thütigkeit der Anniedelungekommission für die Provinzen Weslpreufsen und Posen während der Zoit scit Beginn ihres Bustehens his ultimo 1895 berichtet. Nie 'Thitigkeit genannter Kommission währead des Jahres 1896 sollen nuchfolgende Zeilen schildern.

Im Jahre 1896 sind der Kommission zum freihäıdigen Ankaufe 81 Güter und 27 bïuorliche Grundstiicke angeboten word.n und zwar 25 Guiter und 18 Busernwirtschaften aus polnischer und 56 Giiter und 9 Bauernwirtschaften aus deutechor Hand. Als für die Ansiedelungs\% wocko goeignet wurd'n 7 pröfsere Güter in der Grölse von zusammon 3519,85 ha in Wege des fruiliindigen Ankaufs erworben, so dafs innerhalb der vortlossenen zolin Jahre, wiihrond welcher die Ansiedolungekomininaion in 'Thaitigkeit ist, im ganzeu 148 Güler mit einem Areal von 91331 ha und einem Grundeteuerreinertrage von $781087,04 \mathrm{M}$. angekauft sind. Baucruwirtschaften wurdeu im Jahre 1896 nicht orworben. Somit betrïgt unter Hinzureohnung des in den Vorjaliren angekauften bäuerlichen Areals das Gesamtareal, welches bis Ende 1896 durch die Ansiedelungakommission gelsauft ist, 92724 ha mit einem Grundsteuerreinertrage von 796 063, I6 I. In lländen der Ansiedelungekommission befinden sich demnnch vom $\mathrm{Ge}$ namtareal der Provinz l'osen 2,33 und von der Provinz Westproufuen 0,99 Proz., ron beiden Provinzen zusammen 1,70 Proz.

Von den 7 im Jahro 1896 erworbenen Gütern kamed 3 aus polnischer und 4 aus deutecher Haud.

An Kaufgeldorn wurden 18962282610 M. aufgervendet, so dafs von 1886 bis 1896 insgenamt 56159196,87 M. für Landerwerb auggegoben sind. Im Jahre 1896 stelite sich der gezahlte Erwerbspreis für I ha suf rund $648 \mathrm{M}$., wälirend der Durchschnittapreis für sämtliche erworbenen liegengchaften sich nur auf $606 \mathrm{M}$. für 1 ba beläuft. Die angelegten Preise schwankten zwischen dem 51 fachen und dem 94fachen Gruudsteuerreinertrage.

Der Stand der Besielelung auf don eämtlichen erworbenen Liegenschaften war nun Endo 1896 folgender: 
Der planmärkigen Aufteiluug sind bis Ende 189606066 lan untorworfen worden. Gegen Linde 1895 ist also oin Fortschritt von 7210 ha zu verzeichnen. Rechnet man hierzu dio ohne besonderen Besiedelungsplan, wie die erworbenen Bauerngüter, vergebenen Grundatücke in der Gröleo ron $1046 \mathrm{ha}$, fornor 5 neue Besiedolungaplïno von Gütorn, wolches im Fribjahr 1897 zur Auslegung kommen sollten, mit 3239 ha, so hit gíe zur Auslegung gestellte Fläcbe am 1. April 189770349 ba oder 77 l'roz. der Gesamlorwerbung umfafst. Domnach bleibon in der Vorberoilungeperiode vor dem Besiedelungevorgang 23 Proz. des Uesamtgrunderwerbs.

Die planmalsige Aufteilung jener 66065 ha hat auf Grund vou 110 Hesiedelungeplënen stattgefunden. Die Feststellung der fiskalischen Schadloshaltung iat in diesen T'eilungeplänon derartig erfolgt, dafa von den erwittelien Anreshnungewerten entrichtet werden:

$$
\begin{aligned}
& 3 \text { Proz. in } 76 \text { Fällen, } 2 \% \text { Proz. in } 3 \text { Fillen, }
\end{aligned}
$$

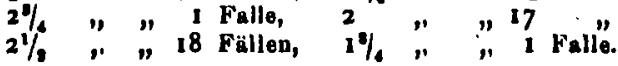

Von den im Jahre 1896 aufgeteilten Gütern sind 9 mit 3 Proz. und nur oins mit 21/2 Proz. Rente, bezw. Pachtsohilling, von den Anreohnungswerten des Orund und Bodens belastet.

Plnnmäfsig ausgelegte Ansiedelerstellen unter Berïcksichtigung der beim Vergebungegesoluäft orfolgten Btellenzusammenlegung und Toilung gab es Endo 18963071 . Dio Gröfse derselben hält sich bei

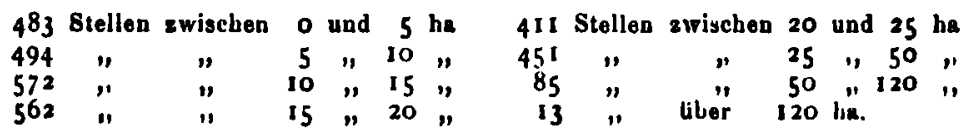

Die Gesamtlä̈che dieser Btellon betriigt $54193 \mathrm{ha}$ im Werte von 35875176 M. Davon waren bis Ende 18962040 Stellen in der Gröfso von 35330 ha und einem Werte von $22291426 \mathrm{M}$. an Aneiedler begebon. Die Grölse dieser Stellon hielt sioh

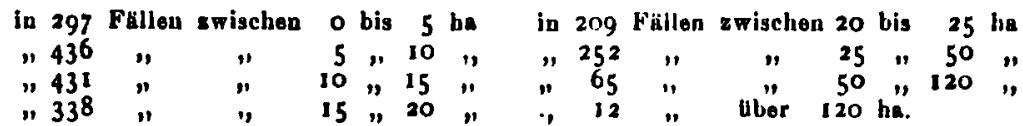

Davon waren zu Pacht oder ale Renteugüter 1976 Btellen vorgeben. Dis Grölse hielt sioh

in 252 Fallon ewischen 0 bis 5 ba in 207 Fällen zwischen 20 bie 25 ha

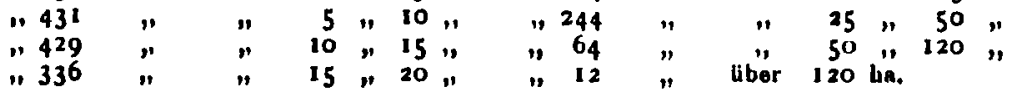

Zu freiem Eigentum verkauft waren 65 Btelien, welche sich

in 45 Fallen in der Grölse zwischon 0 bis 5 ha

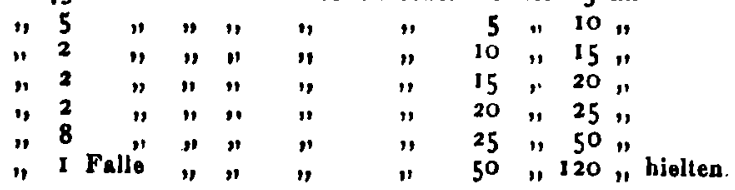


Die Gesnmtgröfso der l'ncht und Rentenstellen betrug $34689 \mathrm{ha}$ im Worte von 22129661 M., dio der zu freiom Eigentum verkuufton Stollon $640 \mathrm{ha}$ im Worte von $161764 \mathrm{H}$.

Wird nun in Rechnung gezogen, dafo dio Landdotalionen fiir öffentlicho Zwecke reichlich 5 Proz. des Stellenaroals nuenachen, no ist das vergebeno Isand ausechliefolioh des verkanfen auf rund 36420 ha zu schätzen. Das sind otwa 39,7 Proz. des Gesamtareals ron 92724 ha. Dio übrigen 60,3 l'roz. den bishorigen Krwerbes stehen noch zur lesiorle. lung zur Verlïgung.

und $\mathrm{zwar}$

Am Bchlusse des Betriebsjahres 1896 blieben 1031 Stellen unbegeben

186 in der Gröfse zwischeu o bis 5 ha, 202 in der Gröfse zwischen 2or bis 25 ha

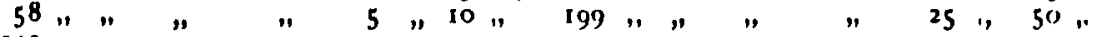

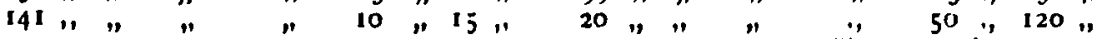
$224 ", \quad " 10 " 15 \%$ " $"$ "

Von den 1975 Ansiedlern, also ausschliefelich derjenigen, welche die Ciuter in freiem Kaufe erworbon haben, waren 131 katholischer und 1844 erangelischer Konfessiou. Die Heimatsprovinz war

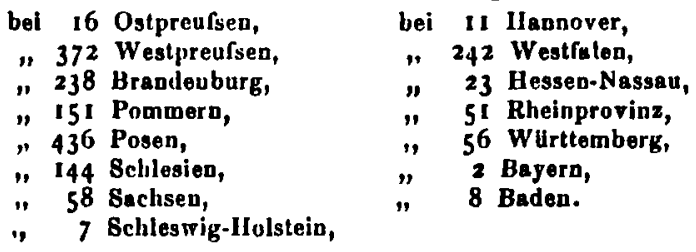

BI Ansiedler waren russische Rïckwanderer und 79 gehörten anderen niclit oben aufgefïhrten deutschen Lündern an. Die Gesamtansiedlerbevölkerung wird auf 10000 Personen geschätzt. Bie wohnen zu 97 Proz. in reschlosenenen Massen angesetzi, so dafs sie in der Lage sind, ein ihron Gowolınheiten entaprechendes kommunules Leben zu entwickeln. Bis uuf ochr wenige Auenabmen ist biuorliche Lebenehaltung die Regel.

Der Verkehr mit Ansiedelungglustigen hat eich während des Borichtsjahree bei dem Zentralbürean zu Posen auf der Höhe der Vorjahre geLalten. Auf den Ansiedelungen selbst scheint der Verkehr von Ansiedelungelustigen stïrkor gewesen zu sein, als in den Vorjahren.

l)azu mag bejgetragen haben :

1) dio gröferere Aufmerksankoit, die dio öffentlicho Presse soit jüngster Zeit den Ansiedelungen schenkt, zumal dadurch, dals sie ihre Berichterstatter die Ansiedelungen beroisen lärst;

2) die Bezichungen, die einzolno Gutsverwalter der Ansiedelungskommission mit den Kroisen, aus denen sio zumeist das A nsiedlermaterial hezicben, dadurch angekuijpft liaben, dafo sie diese Gegenden perönlich brsucht und dort nach corhergegangener Anmeldung bei den Behörden Vertriige über die $\Lambda$ nsiedelung gehalten haben;

3) die Anknijplung von Beziehungen zu der büuerlichen Bevölkeruvg des Westens durch die diesseitige Bestellung von Vertrauenemännern im Finrerstiindnig mit den Kreislandrüten. Dabei war namentlich der Wunsoh mufsgebend, die Kenntnis des östlichen Ansicdelungswesens auch 
in gewise Gegenden mit gesunden bäuerlichen (nicht industriellen) Verhältniseen, wie Hennover und 8chleswig-Holstein, zu tragen, wo sie bisher gänzlich fohlte. Es ist nämlich oino charakteristische Erscheinung, dafs, mangels irgend einer reklameartigen Thütigkeit der Ansiedelungøkommieoion, bieher - nur wenige kleine Bezirke an der Gestaltung der aus dem Westen kommenden $\Delta$ neipdler beteiligt waren. So die niederrheinisohen Kreise Kleve und Hees, die westfälischen Kreise Warburg, Hörter, sowie Miuden, Herford, Bielefeld nebst dem angrenzendep Fürotentum LippoDetmold und einige andere. Mehr oder minder zufallig sind offenbar die ersten Vorbindungen nit diesen Bezirken entstunden. Dann babon glinatige Berichte der Vorangegangenen Varwaudle und Bekanote nachgezoren und daraus hat eich echliefelich ein einigermafsen stïndiger Zuzug entwickelt. Dagegen sind weite andere Gebiete Westdeutsohlands ron dieser Bewegung noch vollig unberlihrt.

E: jot zu hoffen, dafs die Bemühungen, auch hier mehr Anschlufs zu gewinnen, sich bereits im nächsten Jahre in einer 8toigerung der Zahl der Stellenvergebungen bemerkbar machen werden. Immerhiu steht einem Erfolge nach dieser Richtung das in der That auffallend starke Mifotrauen der woetfälischen bäuerlichon Berolkerung gegen die Exietenzbedingungen in den Ländern des Ostens, sowie auch der Umstand onlgegen, dafe nach der Praxis der Ansiedelungskommiseion die Voraussetzung für ein gedeihliches Fortkommen des Ansiedlers ein immerhin nicht unerbebliohes Vermögen iet, das sorgfaltig gohlitet und ungern in nioht ganz sicher erscheinender Anlage oufo Spiel gesetzt wird.

In dieser Hinsicht von der seitherigen Praxis abzulassen, womit sich selbstverständlich der Kreis der Ansiedler erheblich erweitern würde, kuwn nach den anfïnglich gemachten Erfahrungeu und den Beobachtungen, zu denou neuerdings Privatparzellierungen $\Delta$ olafs bieten, nicht in Frage kommen; es sei denn, dafs man sich von vornherein auf erhebliche fiskalische Opfer gefafst machen wollte. Es ist daher auch au eine auegiebige Verwendung wenig bemittelter ländlicher Tagearhuiter aus dem westlichen Deutschland als Aneicdler nicht zu denken. Diese kleinen Vermögen reichen nicht hiu, um die Gefabren des selbständigen Butriebes eiıer bäuerlichen Nahrung zu bestehen, und die kleine Arbeitsstelle, wie sie das Gesetz rom 26. April 1886 vorsieht, ist nicht verführerisch genug, uw zur A uswanderung nach den Ansiedelungoprovinzen Veranlassung zu geben. Der weatdoutsche Tagearbeiter zieht eben nicht nach dem Ostr' $n$, um hier auf eincr Tagenrbeiterstelle mit ländlicher Tagearbeit sein Leben zu fristen, sondern or thut es nur dann, wenn or ein Grundetäck erwerben kann, ron dem er, ohne A rbeitsverdienst aufuchen zu muissen, lehen kann. Das ist aber nur möglich, wenn ein Kapitalvermögen von $2000-3000$ M. vorhanden ist.

Die wirtechaftliche Lage dor Ansiedler zeigt gegen die Forjahre eine wesentliche Beserrung. Dies günstigere Ergebnis ist huupteächlich der besseren Ernte des Jahres $1896 \mathrm{zu}$ dauken, wio deun überlaupt der Eruteausfull die Zahlungsfähigkeit der Aveicdler auffullend stark beeinflufst. Es gilt dies namontlich von denjeuigen Ausiedlern, die sich in den ersten Jabren der Rentezablung befinden. Trotz des geforderten Vermögensnuchwijes bei der Oebernahme und der fiokalischen Ergänzungedarlehen 
nimmt offenbar ein grofser Teil der Ansiedler für die erstmalige Einrichtung seiner Btelle noch in ziemlich erheblichem Mafse den privaten Perconalkredit, insbesondere das gefährliohe Abzahlungegesohäft bei Maschinen und dergl. in Anepruch. Bjo diese Bchulden daun abgezahlt sind und neben der Rente und dem Unterbalt der Familie bare Eraparniese aus der Stelle herauszuwirtachnften ind, befledet sich der Ansiedler in einer Art von Krieis, die sich mit typiecher Kegelmäreigkeit bei den meisten Kolonien wiederholt und in Bezug auf die Gewibrung ron Stundungen und sonstigen Hilfen mit Wohlwollen behandelt sein will. Längere Beobaohtung und eingohende Erkundigungen lassen annehmon, dafs im allgemeinen auch in diescr Periode eine erhoblicho Bparthätigkeit stattfindet, die allerdinge nicht sowohl in der Aneammlung barer Mittel, ale vielmehr in der Abstofsung lästiger Bohulden zu bestehen pflegt.

In einigen älteren Ausierllungen, wo diese Krisis glücklich überwunden ist, scheinen sich die wirtschaftlichen Verhältnisse in erfreulicher Weise zu befestigen. Unter anderom wurde im Berichtsjahre beobachtet, dafs eine Ansiedelung, der vor wenigen Jahren die benachbarten Grundbesitzer die sehleohteste Prognose stellton, sich jetzt in nicht unerheblichem Mafse durch Aktienerwerb an eider Aktienzuckerfabrik hat beteiligen können und allgemein als in guter Wohlstandsentwickelung befindlich bexeichnet wird.

In anderen Ansiodelungen macht sich die Besserung der Lage auch wohl dadurch bemerklich, dafs oin Teil der Ansiedler, nachdem sie tiber die daraus eutstandenen Schwierigkeiten glïcklioh hinaus sind, offen das Geständnis nachen, dafs sie das bei der Niederlassung von ihnen angegebene und eingezahlte Vermögen zum Tuil gar nicht als ihr eigenes besessev, sondern vur von Verwandten entlichen haben, denen sie schon in den Freijahren orhebliche Rückzahlungen haben maohen können. Dafe Ansiedler sich nicht behaupten könven und die besetzte Stelle wieder aufgeben müssen, kommt nur vereinzelt vor. Die Zahl dor verfehlten Besiedelungen hat sich im Berichtsjahr um 3 vermehrt. Von Haus aus unzulänglicho Nittel, Untüchtigkeit im Beruf, unordentlicher Lebenewandel sind in der Kogel die Ursachen des wirtschaftlichen Niedergangs, der schliefelich zum gäurlichen Vermögeusverfall fïhrt.

Die Organisatiou der Ansiedler zu Genossensehaften und Verbänden hat in dem Burichtrjahre weitere Fortschritto gemacht. Kaiffeisen'sche Spar- und Darlehnskassenverejue sind in gröfscrer Anzahl neu begründet worden, wobei die Ansiedler durch Beihilfen zur Anschaffung der Geschältsbuicher und Geldichränke aus dem Dispositionsfond des Präsidenten der Ansiedelungskommission unturstulzt worden sind.

Drain:igegevossenechaften zur Unterhaltung dor von der fiskalischen Verwaltung ausgeführten Draiuagen sind in 8 Ortschaften teils schon begründet, teils noch in der Begründung begriffen.

Erfreuliche Fortschrilte zeigt auch das Molkereigenossenschaftswesen. Einzeloc Ansiedelungen Laben sich schon bestehenden Genossensohaften ange日chlossen, andere haben solche neu begründet.

Wir huben hier im wesentlichen einen Oeberblick darüber gegeben, in wolchem Umfange bis jelzt die Besiedelung durchgeführt ist, und wio 
sich die wirtsohuftliche Lago der Ansiedler gestaltet hat; in nachfolgenden oll nun auf die Thälipkeit der Ansiedelungikommission, welche sie in besondercn auf dem angekauften Areul entwickelt hat, eingegangen werden.

In der zwischenzeitlichen Verwaltung durch die Anoiedelung8kommission befanden wich im Wirtschaftejahr 1895/96 94 Güter mit einem Areal von 80942 ha gegen 90 Verwaltungen des Vorjubres mit einem Flächeninhelt von 75531 ha. Mitenthalteu sind in diesen Ländereien viele Grundatücke, weleho bereite von Ansiedlern benutzt werden.

Von genannten 94 Güterd waren 23 uit einem Gesamtareal von 19377 he erst in den letzten 2 Jahren orworben und befanden sich noch im grofawirtachaftlichen Betriebe. Dieselben erhielten aus der liegierungshuuplkasse einon Zuschufs von $820974,89 \mathrm{M}$. und lieferten an diestlbe $563849,86 \mathrm{M}$. ab, o dafs cin thatsächlicher Aufwaud von $257125,03 \mathrm{~N}$. oder auf den Heklar 13,27 11 . aus dem Assiedelungsfonde notweudig war. Ueberechüsse wurden our in eidzelnen Fiallen erzielt. Die Aufwendungen sind haupteächlich lür Inventarvermehrung gemacht worden. Dieselben orklären sich aus dem beruntergewirtachafteten Zustande, in dem die mojetev Güter übernommen worden.

Aufserdem befanden aich noch fernere 15 Guter mit einem Gesamtareal von 12865 ha im grofswirtachaftlichen Betriebe. Dieselben oind jedoch schon länger als 2 Jahre in fiskalischer Verwaltung und weisen daher auch hinejohtlich ihrer Hentabilität ein günstigeres Resultut als jeno oroteren auf. Von der Regierungshauptkasse erhielten sie einen Zuschufs von 399049,94 M. Sio lieferten an dieselbe $415689,27 \mathrm{M}$, ab, so dafo sioh oin Uoherschufs von $16639,33 \mathrm{M}$. ergab. Solche Ueberschüsse wurded auf 9 Gütern erziclt, wiihrend eich bei 6 Gütern noch oin Minus orgab.

Fon jenen oben genunnten 94 Gülern waren ferner 40 mit einem Gesantareal ron 96922 he in der Besiedclung begriffen.

Diese erhielten aus der hegierungshauptkasse $1041725,63 \mathrm{M}$. und lieferten $88.5023,30$ M. ab, so dafs sie einen Gesamtzuschufs ron $156692,93 \mathrm{M}$. beanspruchten.

Auf 16 Gütern wurdo die Hesiedelung durchgeführt und der grofswirtschaftliche Betrieb aulgelöst. Sie standen jedoch noch unter fiskalischer Verwaltung weil entweder die Gemeindebildung auf Schwierigkeiten gestofsen oder aus anderen Gründen die Auflösung der Gutakase noch hinausgeschoben ist. Es erhielten diese Güter aus der Regierungshauptkasse $17303,82 \mathrm{M}$. und lieferten $4636,83 \mathrm{M}$. ab, so dafs sich ein Zuschufs von 12666,99 M. ergiebt. Derselbe wurde fust nur durch Aufwendungen öffentlich-rochtlicher Natur, wio Kreis- und Gemeindeabgaben, Bauaulwondungen für Armenbïuser und dergl, bedingt.

Insgesamt sind für zwischenzeitliche Verwaltung der Güter seit Erlafs dou Gesetzen 5930 332,25 M. aufgewondet worden, welcher Summo Absobreibungen gegenïberstehen von 1297094,19 M., so dufe sich ein Netioaufwand von 4633238,06 M. ergiebt.

Auch die Meliorationen sind auf den Ausiedelungsgütern weiter gefördert worden. Unter endgiltiger Feststellung der Kosten sind auf 81 Besitzungen zur Gesamtfäche von 45284 ba Drainagen im Oufange von $17402 \mathrm{ha}$ 
ahgenommen worden. Das beträgt rund 38 Proz. des beteiligten A reals. Jer Koslenaufwand fir diese Drninagen beläuft aich auf $3026029 \mathrm{M}$., also hat 1 ha Drainage rund $174 \mathrm{M}$. gekostet. Ausgefuihrt, aber noch niclit abgerechnet sind auf 30 Gütern zur Gesemtfläohe von 20143 hs Drainagen im Umfange ron 7574 ha, d. i. rund 38 Proz. des Areals, zum Kostenansohlage ron $1240000 \mathrm{M}$. In der Ausfuhrung begriffen sind auf 6 Besitzungen zur Gesamilläche von 5653 ha Irainagen im Umfange von 1205 ha, d. i. 21 Proz. des Areale, zum Kostenanschlage von $188200 \mathrm{M}$. Vorarbeiten sind auf 3 Gütern zur Gesamtfläche von 1436 he eingeleitet. A uf diesen werden voraussichtlich 1000 ha oder 70 Proz. mit einem Aufwand von $175000 \mathrm{M}$. zu draiojeren nein. Also beträgt der Gesamtumfang der auggeführten und eingeleiteten Drainagen auf 120 Besitzungen zu 72516 ha Gesam1fiäche 27181 ha Anlagen zum roraussichtlichon Kostenbetrage ron 4629522 M. Das Arbeitspensum des Jahres 1896 betrug 1650 ha gegen 2400 ha des Vorjahres.

Die günstigen Erträge, wolche in den Vorjahren auf den bisher auggeführteu Wiesen- und Noorkulturen erzielt sind, haben auch im Beriohtsjubre durchweg angehalten. Bisher

waren im ganzen fertig Anlagen im Umfauge von 1090 hs
in der Ausfubrung begriffon sind reiter
Vorarbeiten sind ausgefuhrt fur die Anlagen von $200 "$

Auch der Bau ron öfentlichen Wegen wird ron der Kommission oowohl, wie von den Ansiedlern guförtert.

Uebor dio Einrichtung von Viehdepots sweck. Hebung der Rindviehzucht auf den Ansiedelungsgutern batten wir schon im Vorjahre berichtet. Im Berichte für 1896 hejfst es ijber dieselben, defo die Antríge der Ansiedler auf Ueberlassung von Nutzvieb aus demselben aufserordentlich zahlrejch eingehen, oo dafs gie bei weitem nicht alle berücksichtigt werden konnen und auf diejenigen Fälle beschränkt werden müssen, bei denen es sich um Unterstützung vorübergehend bedïrftiger A neiedler durch Verkauf von Vieh auf Abschlagszahlungen handelt. Bisher sind im ganzen 472 Küho und Färsen für einon Gesamtpreie von $95263,50 \mathrm{M}$. an Ansiedlor verkauft worden, wovon 100 Stück im Werte ron $20836,70 \mathrm{M}$. auf dus Berichtsjahr fallen. Gezahlt siod auf die Kaufpreise im ganzen $71474,25 \mathrm{M}$.

Auch ist für die Anpflanzung von Obstbäumen auf den Ansiedelungsgütern weiter Sorge getragen worden. Bei den von Rentegangiedlern bestellten Iäumen übernimmt der Fiskus ${ }^{3} / 4$ der Anschaffungokosten, währond die Kosten der tiur die Pachtatellen oder dje Dotationsländeroien bestimmten Bäumed gauz aus fiskalischen Fonds bestritten werden.

Im Höchstfalle werden 40 Bäume für oine Btelle in zwoi Jahreslieferungen gegeben.

Im Jahre 1896 wurden 6031 Bäume bestellt, ron denen jedoch infolge Rücktritts einer mit der Lieferung von rund 2000 Bäumen betrauten Firma nur 4089 Bäume zum Durchsohnittspreise ron 1,16 M. geliefert worden sind.

Eine umfangreiche Untersuchung des Eigenaufbnues der Ansiedler ist der Denkschrift für 1896 beigefügt. Auf die Einzelheiton derselben 
hier einzugehen, würde zu weit fiihren. Es sei nur orwähnt, dafe in den ersten Jahren der Thütigkeit der Ansiodelungakommission dieselhe von dem Acsichtspunkte ausging, dafs dio fijr die Ansiedler bestimmten privatwirtschaftlichen Gchäudo vor dor Begobung rom Fiskus hergcstellt werden müfalen. Mit diescn Banausfiihrungon hatlo die Kommission jedoch keiu Glïck. Vielfach sagten dieselbon din A nciedlern nicht zu. Dahor wurde in den Jabren 1893-1895 die Thütigkeit dor Kornmiasion nuch dieser Richtung hin eingeschrănkt. Im Jahre 1896 hat man jedoch wicder mit deil Aufhau ron Ansiadolungagehöfton in umfangreichem Mafsetabe hegonuen. Es irt dies geschehen, woil man glaubt, jutyt infolge sorgliiltiger Brobachtung der Ansiedler bri den ron ihnen in Bjgenbau aufgeführten Ilöfon dio friuher gemanhton Fohler rormeiden zu kiinmon.

ITa nü sohlicfslich den Ansiedelungkfonds anbetrift, so haben dio (Gosmtausgaben

bis zum 1. April 1896 betragen

Dem stebt eine Einnalıme gegeniiber von

Mithin betragen die thatsärbliclien Auggalien
$80892522,30 \mathrm{M}$.

10970732,95,

$69921789.35 \mathrm{M}$.

Eg bloibt demnach noch eine Summe ron 90078210,65 M. zur Fortsetzung der Besicdelung tibrig.

Zum Schlufs sei nun noch der Finfübrung dos A nerbenrechts auf den Ansiedelungastellen gedacht. Mit dem 1. Oktoher 1896 ist das Gesetz rom 8. Juni 1896 über das Anerbenrecht bei llonten- uod Ansiedelungegïloru in Kraft getreten. desen Brstimunungen unch dis auf Grund des Gesetzes rom 26. April 1886 zu Eigentum rergebenen Stellen unterworfen sind. Gemüfs \$ 2 des Gesetzes sind simtliclie liggentiimor ron A nsiednlungsetrllen dariiher gehört worden, ob sio mit der lintragung dor A norbengutnoigennachaft in das Grundhuch einverstanden seion. Nur ninige Widerapriiche huben sich danegen orhobon, die Mehrzahl hat sich damit oinverstandon rrklürt. Die Verwoigerungen dor Piintragung hepehtüinken sich nun nirht auf die beidon im Gesetz alluin zugelnesenon Griinde, niimlich dio mangelndo wirtachaftliche Selbetändigkeit oder dns Vorwiegan gemeinwirtachaftliehor Interessen gegen dio Aufrechtorhaltung dirser Sclhetändigkeit, sondern sie richton sich zum Toil auch gegen die Tendinz des Gevetzes, und kommen in der Behauptung zum Augdruck, dafis durch die Verfügungsbeschrïnkungen des Gosetzer die Möglichkeit der Woiterv"rianferung hepintrïehtigt und dadureh der Kaufwert der Strllen herabgodrückt worde. Dafs dies Wideretreben weniger nuf Verstiindnis des Inhaltes des Gesetzes als auf rinem allgemeinen Mifsbehagen gerenüber einem angoblich geülten gesetzlichen $\mathrm{Zwange} \mathrm{beruht,} \mathrm{orgiebt} \mathrm{sich} \mathrm{schon} \mathrm{daraus,} \mathrm{dals} \mathrm{dor} \mathrm{von} \mathrm{den} \mathrm{bo-}$ treffenden Ansiedlern in froier Vercinbarung unterzeichnete Reutengutsvertrug bereits dieselben und viel writergihende lioschrünkungen dor Veriiufserungsfreiheit onthält als die des Gogetzes.

Soweit Widersprüche nicht orhoben sind, ist dio Nintragung der Anrrbengutaeigenechaft im Grundhueh hereils horbrigofiibrt. Hiermit wird vach Erlcdigung der Eingpriiche weitor fortgefuturen werden. 